\title{
Philosophiques
}

\section{Politiser ou privatiser l'ethnie ? Réflexion à propos du bien commun en Afrique postcoloniale}

\section{Essodina BAMAZE N'GANI}

Volume 45, numéro 2, automne 2018

URI : https://id.erudit.org/iderudit/1055270ar

DOI : https://doi.org/10.7202/1055270ar

Aller au sommaire du numéro

\section{Éditeur(s)}

Société de philosophie du Québec

\section{ISSN}

0316-2923 (imprimé)

1492-1391 (numérique)

Découvrir la revue

Citer cet article

BAMAZE N'GANI, E. (2018). Politiser ou privatiser l'ethnie ? Réflexion à propos du bien commun en Afrique postcoloniale. Philosophiques, 45(2), 419-444. https://doi.org/10.7202/1055270ar

\section{Résumé de l'article}

En raison de fortes tensions ethniques qui le mettent en branle, l'État en Afrique vit un délitement du tissu collectif. Dans un contexte de pluralisme ethnique fort, la négation de ce pluralisme par la politique d'assimilation a eu pour effet contraire l'édification d'un sectarisme ethnique donnant lieu à une instabilité socio-politique. En réaction, la prise en considération institutionnelle de l'ethnie n'a pu empêcher cette instabilité. Oscillant ainsi entre politique d'assimilation et naturalisation normative de l'ethnie, l'État y vit une crise du bien commun. Le présent article vise à montrer que la gestion du pluralisme ethnique en Afrique ne saurait se réduire à de simples dispositions politico-juridiques. Il s'agit bien plutôt de promouvoir la perspective de la postcommunauté qui vient fédérer trois types de mobilisations enchevêtrées : l'une relative à la conscience, l'autre à la participation de toutes les composantes communautaires au bien commun, enfin celle relative aux nouvelles responsabilités de l'État. 


\title{
Politiser ou privatiser l'ethnie? Réflexion à propos du bien commun en Afrique postcoloniale
}

\author{
ESSODINA BAMAZE N'GANI \\ Université de Sorbonne Paris IV (France) \\ essodinabamaze@gmail.com
}

\begin{abstract}
RÉSUMÉ. - En raison de fortes tensions ethniques qui le mettent en branle, l'État en Afrique vit un délitement du tissu collectif. Dans un contexte de pluralisme ethnique fort, la négation de ce pluralisme par la politique d'assimilation a eu pour effet contraire l'édification d'un sectarisme ethnique donnant lieu à une instabilité socio-politique. En réaction, la prise en considération institutionnelle de l'ethnie n'a pu empêcher cette instabilité. Oscillant ainsi entre politique d'assimilation et naturalisation normative de l'ethnie, l'État y vit une crise du bien commun. Le présent article vise à montrer que la gestion du pluralisme ethnique en Afrique ne saurait se réduire à de simples dispositions politicojuridiques. II s'agit bien plutôt de promouvoir la perspective de la postcommunauté qui vient fédérer trois types de mobilisations enchevêtrées: l'une relative à la conscience, l'autre à la participation de toutes les composantes communautaires au bien commun, enfin celle relative aux nouvelles responsabilités de l'État.
\end{abstract}

\begin{abstract}
Due to high ethnic tensions at play, the State in Africa is witnessing a disintegration of the social fabric. Against the background of a pervasive ethnic pluralism, the weakening of this pluralism through the policy of assimilation had the unwanted effect of establishing ethnic sectarianism, culminating into social and political disruptions. Subsequently, the institutional consideration of ethnicity could not prevent this instability. Torn between the policy of assimilation and the normative naturalisation of ethnicity, the State is undergoing a crisis in terms of the common good. This paper seeks to demonstrate that the management of ethnic pluralism in Africa cannot be watered down to simple political and legal provisions. It has far more to do with promoting the perspective of post-community which merges three types of interrelated concepts: one related to conscience, the other to the involvement of all community components working for the common good, and lastly the one dealing with new duties of the State.
\end{abstract}

\section{Introduction}

La question ethnique apparaît en Afrique postcoloniale sous une quadruple dimension: tantôt elle apparaît comme la réalité africaine fondamentale, tantôt comme la cause de l'échec de l'État-nation, tantôt comme une fiction idéologique, tantôt comme la base d'une solution des problèmes politiques africains. À la faveur de ce contexte, "politiser ou privatiser l'ethnie?» donne du lustre à une autre préoccupation: celle de la place des ethnies dans la gestion du bien commun en Afrique postcoloniale. Notion centrale dans la conduite des affaires humaines, le bien commun y est mis à l'épreuve par de nombreuses tensions ethniques symbolisant un passage "épineux» des 
sociétés traditionnelles marquées par la prégnance de la «solidarité mécanique " aux sociétés modernes dites "organiques». Il en va d'une crise dans la gestion du bien commun. En effet, le citoyen africain à l'ère postcoloniale n'a pas fondamentalement abandonné une manière de concevoir le bien commun en fonction de présupposés conjointement «mécanique » et anthropologique. Il appert que ce dernier est resté redevable au postulat d'après lequel lorsqu'un membre d'une "ethnie " accède au pouvoir, c'est tout le creuset ethnique qui se trouve promu: comment en effet comprendre autrement l'attitude des citoyens affirmant leur allégeance inconditionnelle au chef de l'État en Afrique chaque fois qu'un des leurs est nommé à un poste ministériel?

Qui plus est, selon cette perspective, "un ministre ou un haut fonctionnaire n'occuperait un poste dans l'administration de l'État qu'à titre de converture occultant un service de sa famille, de son village ou de son ethnie, lesquels passeraient pour constituer une "source du droit" bien plus impérieuse que tous les décrets formels de la République» (Dijon, 2009, p. 598). Or, dans sa justification libérale, la séparation de l'État et la famille, pour ne citer que celle-là, avait pour intérêt d'assurer la protection du pouvoir politique en le mettant à l'abri d'une mainmise interne. Car: "L'État n'est pas libre quand le pouvoir est confisqué et exploité par les membres d'une famille [...]» (Walzer, I997, p. 47). Ainsi donc, ce constat appelle la réflexion, notamment lorsque la «solidarité mécanique " toujours à l'œuvre dans le terreau ethnique en vient à forger le sentiment de "ghetto " au sein de l'État en Afrique. Dans cette optique, l'émergence d'un «ethnocentrisme partisan " à l'heure du pluralisme politique en Afrique de même que la pratique du vote ethnique qui a pu s'en réclamer boudent une compréhension du bien commun comme «bien suprême». Comme l'illustre si bien ce proverbe africain: "Quand ton oncle est dans le figuier, tu ne manqueras pas de figues». Ton oncle, assurément! De là, comment faire en sorte que les sept millions de Togolais ou les vingt-quatre millions de Camerounais qui n'ont aucune relation de parenté avec «cet oncle» puissent aussi disposer de figues à satiété?

Il apparaît pour le moins que la solidarité africaine, telle que corroborée par ce proverbe, est une catégorie discursive ${ }^{1}$ du bien commun dans la figure moderne de l'État en Afrique. Idée qui s'explique, entre autres, par une mainmise des dirigeants sur les leviers ethniques, par une large adhésion populaire aux logiques mobilisatrices et calculatrices des identités ethniques, par l'absence du bien commun transcendant tous les particularismes ethniques, également par la crise de l'État postcolonial dont la formation et les mécanismes font état d'une sorte de désétatisation de l'État en Afrique. Réalité que nous souhaitons "dépasser" pour repenser à nouveaux frais les

1. Au sujet de la solidarité africaine comme catégorie discursive du bien, nous renvoyons le lecteur à l'article de Kiamba, 20I I, p. 22-25. 
conditions de possibilité du bien commun dans l'État postcolonial d'Afrique en tant que "bien suprême", c'est-à-dire celui en vertu duquel toutes les communautés ethniques au sein de l'État doivent désormais être constituées. À cette fin, éclairer les ombres et lumières de cette problématique exige en amont que soit clairement appréhendés les contours conceptuels d'une notion a priori controversée: l' "ethnie». Puis établir les préalables d'un discernement social des contrastes liés aussi bien à une politisation qu'à une privatisation des identités ethniques. À ce titre-là, afin d'introduire les soubassements de ce qui nous semble constituer dans la solidarité ethnique la crise du bien commun en Afrique, nous voulons montrer comment cette forme de solidarité donne lieu à une "éclipse» du bien commun au sein de l'État, et comment elle permet de mener une réflexion «contextualisée» en philosophie politique sans pour autant conduire à relativiser l'idéal dont la philosophie est porteuse.

\section{L'indicible «nous»: ethnie versus bien commun}

Par «nous» il faut entendre ici la référence aux valeurs communes, cellesci étant la force motrice de l'unité étatique sans laquelle il n'y aurait non seulement pas d'existence dans la diversité, mais non plus d'État. Du fait que tout État démocratique se compose aujourd'hui d'appartenances multiples, puisqu'il repose sur le présupposé du "pluralisme social ", la quête de bonheur collectif que symbolise en ce point précis le "nous" est inévitablement le fondement existentiel de l'État et appartient donc à son essence. Il est cependant impossible d'entrer ici dans les détails des relations entre le «nous» et les multiples identités que représentent par exemple les ethnies, car il faudrait auparavant savoir ce que c'est qu'une «ethnie».

\subsection{L'ethnie: d'une notion à géométrie variable à un concept}

Le concept, nous dit Kant (2006, p. 3 Io), est un concept de la réflexion, c'est-à-dire "[...] l'état de l'esprit où nous nous disposons en premier lieu à découvrir les conditions subjectives sous lesquelles nous pouvons parvenir à des concepts». Dans ce climat, envisager une conceptualisation de l'ethnie tout en l'enracinant dans un univers socio-politique particulier, notamment celui de l'Afrique, peut paraître comme une «trahison » à l'égard de la philosophie: d'abord parce qu'elle se veut dans son essentialité une réflexion «a priori », «universelle» et "générale », et ensuite parce que l'ethnie n'est pas une réalité purement africaine. À l'analyse toutefois, l'évidence de cette présupposée trahison est émoussée par une formule de Rawls (I993, p. 245) d'après laquelle "Les fins de la philosophie politique dépendent de la société à laquelle elle s'adresse». À ce titre par exemple, Kymlicka ne manqua pas de souligner que son modèle de citoyenneté multiculturelle tire ses repères des problèmes de relations ethnoculturelles tels que posés au sein des démocraties anglo-américaines où, on le verra ultérieurement, l'identification de 
l'ethnie ${ }^{2}$ à des populations homogènes issues de l'immigration n'a rien de commun avec ce qui résonne comme une ethnie en Afrique. Procédant ainsi par cette nouvelle dimension de la philosophie ayant un sens aigu des contextes, nous adopterons donc une démarche ex datis. Selon cette option, nous proposons de procéder à une sorte de "désidéalisation de la philosophie» (pour reprendre l'expression forgée par Renaut), en opérant à travers les données empiriques: c'est-à-dire un certain nombre de données que l'expérience, notamment sociale, désigne par «ethnie» en Afrique.

En conséquence, pour la réflexion que nous proposons dans les lignes qui suivent, la question que soulève cette préoccupation méthodologique est pour nous celle de déterminer ce à quoi correspond jusqu'à présent une ethnie en Afrique. L' «ethnie ${ }^{3}$ » comprise en son sens étymologique comme "peuple» ou comme "nation» est une des composantes les plus manifestes et les plus décriées ${ }^{4}$ de la culture politique de l'Afrique contemporaine. Elle est aussi au cœur de presque tous les débats concernant la démocratisation des États africains. Malgré cette popularité, ou peut-être à cause d'elle, l'ethnie reste problématique. Problématique parce que, pour plus d'un, elle ne correspond à aucune réalité. Ce qui n'est rien d'autre que d'affirmer: l'ethnie n'existe pas. Or, "Si l'ethnie n'existe pas [...] que nous reste-t-il à étudier?» (Amselle et M'Bokolo (dir.), I999 [I985], p. III). C’est l'occasion d'emblée de renvoyer dos à dos deux approches qui manqueraient le sens même de l'«ethnie".

L'une consiste en une "désubstantialisation » de l'ethnie: une logique purement constructiviste de ce qu'on s'accorde à désigner par «ethnie». Certains n'ont pas manqué de mettre en avant le caractère purement «instrumental » de l'ethnie en fondant leur argumentation sur la nature composite de celle-ci. Pour preuve, la parution de l'ethnie Nibolek au Congo. En effet, c'est au cours des élections présidentielles de 1992 que le terme à "consonance ethnisante", Nibolek, sera forgé à partir des initiales des groupes Niari, Bouenza et Lékoumou, pour désigner l'ensemble des régions favorables au candidat Pascal Lissouba (Dorier-Apprill, I997, p. I68). L'ensemble des localités disparates ainsi réunies dans la fabrique de l'«ethnie Nibolek» contribue à renforcer l'évidence que les ethnies ne sont que la résultante de stratégies politiques, donc une simple construction. Mais dans

2. Ce point a été développé dans la première partie du chapitre II datant de 200I, p. 24-44.

3. Inventée dans la littérature française du XIX ${ }^{e}$ siècle pour gommer le terme de race. Cela en la distinguant d'autres formes d'appartenances telles que la région, la religion, la tribu, le clan, etc.

4. Cela d'autant plus que les regroupements ethniques constituent, dans leur expression postcoloniale exacerbée, d'une part, un obstacle à la mise en place de véritables politiques publiques et, d'autre part, la source d'une abondante littérature dont il serait inutile de vouloir répertorier quelques travaux ici dans la mesure où cette éventuelle liste serait très loin de l'exhaustivité. 
le même temps, la formation de cette ethnie de même que son fonctionnement dans le vécu congolais peut participer d'une compréhension de l'ethnie comme une identité dialogique et mettre un terme à la pertinence de cette première approche. Cela d'autant plus que, d'une simple "fabrique", l'ethnie finit par véhiculer chez les populations concernées une certaine manière d'être que vient corroborer une formule de Amselle et M'Bokolo ((dir.), I999 [1985], p. I23): "Si celui que j'interpelle se tourne, c'est qu'il "répond" effectivement à ce nom". Aussi se trouve-t-elle, cette première approche, limitée par l'existence d'un minimum de substrat historique nécessaire à la cristallisation de ce sentiment d'être différent. En un sens, par exemple, on reconnaîtra quand même que l' «invention" d'une ethnie ne saurait être possible sans le sentiment, aussi minimal soit-il, d'être différent: "Les Bamilékés sont dynamiques, avares et rusés"; "Les Bassa sont sauvages et violents "; "Les Béti sont hospitaliers et naïfs", clichés rapportés en note infra-paginale par Mbonda (2003, p. Io). Plus encore, l'idée d'une inadéquation entre l'ethnie et la "réalité » à laquelle elle est censée correspondre ne suffit pas pour dénier l'existence des ethnies en Afrique. Car la «réalité » doit être envisagée sous une double perspective: celle d'abord du monde physique, auquel cas l'on pourrait valider l'inexistence des ethnies en Afrique, et celle ensuite du monde psychologique, auquel cas l'on admettra l'existence des ethnies du moins dans le psychisme des personnes qui s'en réclament ou qui y font une référence constante.

L'autre approche revient à «essentialiser» l'ethnie, à présenter cette forme d'appartenance comme "primordiale"; mieux dit, à en faire un pôle d'homogénéité fondé sur des critères tels que les liens de sang, la culture commune, les affinités naturelles et repérables comme telles dans des espaces géographiques bien délimités. Le paysage ethnique des Bamana nous en donne une ébauche de compréhension: "Leur habitat s'étend du $11^{e}$ au $14^{e}$ degré de latitude nord et $d u 7^{e}$ et $11^{e}$ degré de longitude ouest [...]» (Bazin, I999 [1985], p. I16). D'apparence homogène du point de vue géographique, l'ethnie Bamana ne conserve pas pour autant moins de disparités sur le plan interne. Son apparente homogénéité fut jadis bâtie sur le critère d'une langue commune. Or, à lire par exemple Chrétien et Prunier (I989), et d'une façon générale tous les écrits autour des ethnies en Afrique, on saisit comment la langue bambara est aussi parlée dans la région de Segou ou dans celle de Beleko. De ce constat, la langue et l'espace géographique étant insuffisants pour affirmer l'homogénéité d'une ethnie, on pourrait derechef faire le grief à cette approche essentialisante des ethnies de négliger le caractère fluctuant de tous les groupes sociaux. Bien entendu, pareil reproche supposerait la réaffirmation de la thèse de l'«invention» des ethnies, cela même que récuse à bon droit Otayek (200I, p. I35) en affirmant que, "pour "inventer» une ethnie, il faut qu'il y ait le minimum de substrat historique nécessaire à la cristallisation, d'un sentiment d'être différent". 
Aux confins de ces deux approches, refusant de jeter le bébé avec l'eau de bain et adoptant du même coup une démarche bijective, nous trouvons plus clair de définir l'ethnie comme le sentiment d'appartenir à un groupe humain différent des autres par des critères réels ou supposés. Du reste peuton s'interroger sur le rapport entre ethnie et ethnicité? Sans doute serait-il vain de chercher à opérer une distinction entre «ethnie » et «ethnicité », étant bien entendu que cette distinction ne saurait avoir aucun autre fondement que celui de vouloir remplacer l'ethnie par un autre concept plus expressif: celui de l'ethnicité. Mais toujours est-il que, dans cette démarche, rien ne nous paraît véritablement distinctif entre les deux concepts en dehors de ce souci de vouloir remplacer un terme "fourre-tout» (Weber, 2003, p. I39) que représente l'ethnie par un autre beaucoup plus significatif. Pour notre part, nous souscrivons entièrement à la thèse d'Amselle (I990) d'après qui ethnie et ethnicité entretiennent une complémentarité fonctionnelle.

Reconsidérer l'ethnie comme sentiment d'appartenir à un groupe humain différent des autres par des critères réels ou supposés ne fait aucunement obstacle à l'être-ensemble politique, puisque ce sentiment prend origine dans l'immédiateté de l'entendement humain. Par exemple, se sentir Bamiléké ou Béti n'a rien d'effarant. C'est, comme le dirait Taylor (I994, p. 42), «[...] un besoin humain vital». Vital en ce sens que tout être humain vient au monde dans une communauté spécifique dont le patrimoine communautaire contribue d'un poids énorme à la formation de sa personnalité de base. De ce fait, la personnalité de tout être humain apparaît comme le résultat d'une matrice communautaire. En ce sens, prétendre le «sevrer» de ce patrimoine serait identique à une éventuelle demande adressée à quiconque perché sur une branche de la scier lui-même. Et comme nul, même de nature adamique, ne saurait élaguer une branche à laquelle il se trouve juché, on comprend aisément dans ces conditions que la conscience ethnique puisse se dresser dans une certaine mesure contre les valeurs suprêmes (de l'unité nationale et de l'intérêt général) auxquelles chaque citoyen doit participer par son activité individuelle ou par l'action des différentes collectivités en vue de donner un sens et une existence dans un cadre plus vaste, celui de l'État. À bon escient, ce sentiment en vient à faire obstacle à partir du moment où il sert de prototype d'évaluation de tous les rapports sociaux. Ainsi, comment, dans un pays composé de deux cent cinquante ethnies à l'instar du Cameroun, envisager le bien commun?

\subsection{Le bien commun comme défi de la pluralité ethnique}

Si la référence au bien commun se trouve inscrite dans chaque association humaine comme un "patrimoine commun", il n'en demeure pas moins vrai que sa définition reste problématique comme d'ailleurs il en est de toutes les notions usitées. Le bien commun, que l'on est en droit de considérer comme une valeur inhérente à toute association humaine, recouvre plusieurs significations qui se ramènent pour l'essentiel à deux: «biens 
communs » au pluriel et «bien commun » au singulier. À en croire Mbonda (20II, p. II3):

Dans sa première acception, on parle de «biens communs» (au pluriel) pour désigner un certain nombre de ressources telles que l'air, l'eau, la terre [...] la paix ou la justice, la dignité humaine, le respect de la vie... considérées habituellement comme l'objet d'une aspiration universelle et comme des biens fondamentaux pour tout être humain. Dans sa seconde acception, le bien commun (au singulier) désigne plutôt une valeur supérieure, transcendante par rapport aux individus, et fixant pour tout être humain l'horizon de l'accomplissement de soi.

Dans le bref exercice que nous envisageons concernant l'intellection du bien commun dans les États modernes en Afrique, nous préférons donc recourir à la deuxième signification (celle au singulier) en tant que cette signification permet de comprendre le bien commun comme un bien suprême, une valeur commune ou encore comme un idéal auquel aspirent les membres d'une même communauté nationale. Cet idéal désigne tout autant la «finalité de la polis» dont le fondement serait ici le bien suprême auquel un processus d'adhésion des différentes communautés organisées elles-mêmes en vue d'un certain bien, dont en guise de dernier exemple, la «théorie de la polis» par laquelle Aristote démontre la nécessité d'une «communauté politique» serait une formule particulièrement explicite. En effet, dans Politiques (Livre I, I983, p. 26), Aristote écrit:

Puisque toute cité, nous le voyons, est une certaine communauté, et que toute communauté a été constituée en vue d'un certain bien, il est manifeste que toutes les communautés visent un certain bien et que, avant tout, c'est le bien suprême entre tous que vise celle qui est suprême entre toutes, c'est-à-dire celle qui contient toutes les autres. Or c'est celle qu'on appelle la cité ou communauté politique.

$\mathrm{Si}$, pour Aristote, le bien suprême en tant que bien commun se laisse clairement identifier au bien vers lequel tendent tous les autres biens particuliers autour desquels se trouvent articulées les différentes communautés particulières, il nous semble alors que l'idée d'une "crise du bien commun » en Afrique trouve ses repères dans le revers du syllogisme aristotélicien: la communauté politique ou cité contient le bien suprême auquel elle aspire et auquel aspirent toutes les autres communautés constituées elles-mêmes en vue d'un certain bien. En conséquence, la communauté politique renferme en elle-même toutes les autres communautés. Or, dans le registre de l'État moderne en Afrique, toutes les communautés ethniques constituées ellesmêmes en vue d'un certain bien s'accaparent de l'État ou du moins tentent de se l'accaparer. Ainsi en vient-on à une crise du bien commun qui apparaît sous deux angles et qui porte à s'interroger véritablement sur ce qu'est ce "bien" qui devrait rester "commun " : d'une part, chaque groupe ethnique utilise l'État à son profit et, d'autre part, investi au sommet de l'État, chaque 
chef met les différents groupes ethniques à son service. Partant, on pourrait formuler deux thèses tout à fait conciliantes: la tendance des chefs d'État à mettre les différents groupes ethniques à leur service a pour conséquence cette logique de "fils du terroir» appelant une identification ethnique en période électorale et la crise de l'État postcolonial d'Afrique. L'attitude de Paul Biya (président du Cameroun) dans les années I99I-I992 pourrait illustrer la tendance des chefs d'État africains à mettre les différents groupes ethniques à leur service:

Confronté en I99I à l'expression publique du mécontentement des Foulbé à l'égard de son régime, le président Paul Biya nomme un des leurs, Sadou Hayatou, au poste de Premier ministre en avril I99I. La défaite électorale du parti au pouvoir dans la province du Nord lors du scrutin de mars 1992 conduit à son limogeage. Afin de se relégitimer dans la province de l'Ouest auprès des Bamileké, en avril 1992 tous les départements «Bamileké» de la province de l'Ouest [...] sont représentés par des ministres et assimilés. À ce don ne répond pas lors des élections présidentielles du I 2 octobre le contredon de suffrages en faveur de Paul Biya. D'où la punition symbolique de l'Ouest sous la forme de la réduction sensible du nombre de ministres de six à deux [...] (Sindjoun, 1998, p. 2r).

Cette incapacité de l'État à imposer sa souveraineté sur le plan national face aux ethnoéthiques qui tentent d'envahir le monopole détenu par l'institution "État ${ }^{5}$ " (voir Mbembe, 2000, p. 4I-93 et p. 95-I38) pourrait également être lue comme une crise de l'État postcolonial en Afrique noire. Il en va, dans l'État moderne en Afrique, d'un «État rhizome ", d'un «État néopatrimonial» ou encore d'une «politique d'affection» suivant les terminologies propres à Bayart (I989, p. 270-280), Médard (I991, p. 323-353) et Sindjoun (r998, 64p).

Cet écartèlement de l'État entre son accaparement par les communautés ethniques d'une part, et sa mise au service des acteurs politiques, d'autre part, semble indiquer qu'il n'y existe pas encore de bien commun au sens de souverain bien, ou que ce bien souverain s'inscrirait dans le registre des choses qui n'ont pas de corps comme en témoigne le nullum subest corpus de Cicéron. En faisant porter l'accent sur cet écartèlement et en indiquant que cet écartèlement éloigne quotidiennement de la sphère de la "chose publique", réexaminons la gestion de la pluralité ethnique en Afrique pour en tirer quelques perspectives de recherche.

5. En ce sens, cette piste d'analyse conjointe pourrait consister à montrer que le problème réside dans la nature-même de l'institution «État» sur le continent africain dans sa difficulté à synthétiser les particularismes communautaires avec l'exigence de neutralité libérale qui caractérise l'État dans les sociétés de type occidental. Nous n'insisterons pas tant sur les détails de cet argument dans le corps de notre texte puisque, dans un rapport d'antériorité, c'est à nos yeux la forte communautarisation de la société qui empêche l'enracinement de l'État au sens wébérien du terme et non l'inverse. 


\section{La conscience ethnique entre politisation et privatisation: le risque de la boîte de pandore}

La trajectoire de la politisation et celle de la privatisation des ethnies doit être analysée sous le prisme dualiste «espace public» par rapport à «espace privé». Une telle entreprise peut paraître rétrograde ou désuète dans la mesure où cette distinction, valable à la naissance de l'État bourgeois, est devenue "factice» aux yeux de Habermas (I986, p. I50) au motif de ce

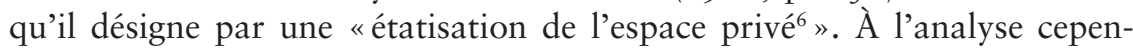
dant, l'évidence de cette restriction s'émousse au regard d'une pratique du pouvoir moderne prisonnière de "l'esprit de cour», d'une sorte de «curialisation des mours politiques ${ }^{7}$ » qui rend épineuse la construction d'un espace public véritable dans les sociétés africaines contemporaines. Ainsi devient-il légitime d'égratigner ce vernis idéologique qui, partant de la persistance de ces liens sociaux primaires, peint l'«État multiethnique " (Tshiyembé, 2007, p. 34I-353) ou la «Nation à polarisation variable» (Nicolas, I987, p. I 57I74) comme un axe majeur d'une démocratie ancrée en Afrique.

\subsection{Qu'est-ce que politiser? La trajectoire de la politisation des ethnies}

Abordant en ce point la question ethnique "dans l'Afrique d'aujourd'hui » et non plus dans l'Afrique coloniale, période de «fabrique» des ethnies ou ethnicités africaines, la trajectoire de la politisation des ethnies doit être nettement distinguée de celle, coloniale, au cours de laquelle la "politisation» de la société faisait référence à une sorte de "dislocation» puis de "hiérarchisation» des différentes ethnies à des fins politiques, économiques et culturelles. Par exemple, au Cameroun, lors de la colonisation, le colon mit en place l'idéologie et la supériorité culturelle des Peubl (groupe minoritaire) à l'égard des Kirdi (groupe majoritaire non islamisé) dont la conséquence fut l'attribution de privilèges au premier groupe. Constatation plus troublante, cette idéologie a été renforcée par le régime postcolonial d'Ahmadou Ahidjo (un Peuhl), puis conservée dans le contexte de démocratisation du Cameroun, période au cours de laquelle cette idéologie a favorisé la production stratégique de la kirditude. Entendre par là: «un terme politique qui reflète une protestation contre l'exclusivisme et la discrimination du côté des musulmans et une prise de conscience croissante que les non-musulmans peuvent représenter une force politique importante s'ils s'unissent ", d'après Ibrahim Mouiché (cité par Akono Evang, 20I4, p. I63). Pris au pied de la lettre, ce sentiment, la kirditude, ainsi que toutes les autres formes de replis identitaires à l'œuvre sur le continent noir invitent à penser les modalités

6. Étatisation qu'il qualifie de société civile et qui pourrait comme telle, voir son analogon dans l'idée d'une "société civile ethnique» dont fait mention Sindjoun (I998, p. 5-7) dans le cadre de sa réflexion sur l'Afrique noire et plus spécifiquement du Cameroun.

7. Terme que nous empruntons volontiers à Bidima, 2000, p. 90-106. 
d'une gestion des différences ethniques autrement que par l'activation de la fibre ethnique.

Dans ce sens, la trajectoire d'une politisation de l'ethnie trouve son origine dans le constat de la pluralité ethnique comme un "fait " indéniable de la société postcoloniale d'Afrique laquelle, de ce fait, vit des conflits ouverts dans certains contextes et des conflits fermés dans d'autres contextes nationaux. En effet, la postcolonie en Afrique est marquée par l'exacerbation des conflits, non plus entre les États, mais entre les différentes identités qui se retrouvent au sein d'un même creuset national. Comme l'a remarqué Mbonda (2003, p. 5) à la suite de Chrétien: "Les ennemis, on ne les trouve pas en dehors des frontières, mais à l'intérieur, et ils sont identifiables non pas à leurs uniformes, mais à leurs appartenances ethniques [...]». Ainsi, la bigarrure ethnique de l'État postcolonial en Afrique suscite une minutieuse réflexion, étant donné les conflits qui ont ensanglanté le continent noir et qui ont révélé leur caractère épouvantable par le nombre élevé de leurs victimes ${ }^{8}$. Dans un pareil univers, c'est-à-dire celui de la prééminence d'une guerre civile, il est impérieux de savoir organiser cette bigarrure pour éviter des conflits ethniques inextinguibles en raison des enjeux de pouvoir et des questions fondamentales d'identité qu'ils mobilisent. C'est à ce titre, qu'intervient une dimension de la politique comme étant une «activité au service de la collectivité ».

En effet, répondant à la question: Qu'est-ce que la politique? Freund (1965, p. I78) fait de la politique un garant de "[...] l'ordre au milieu de luttes qui naissent de la diversité et de la divergence des opinions et des intérêts». En ce sens donc, la question "qu'est-ce que politiser? " trouve son repère dans cette finalité de la politique et du pouvoir politique comme capacité "de rendre possible des modes d'interaction non conflictuelle entre individus et entre groupes, à travers des stratégies de négociation et de compromis mutuellement bénéfiques»(Mbonda, 2003, p. 6). Quelles peuvent être ces stratégies de négociation et de compromis qui se révèlent bénéfiques pour tous? Activité au service de la collectivité, et étant donné sa sphère d'organisation, la politique se révèle être au service de l'homme et des différentes identités auxquelles ce dernier s'identifie dans un État. Ce pourquoi, par exemple, la thématique de la «diversité» dans la politique française aura permis désormais la représentation des "minorités visibles» dans l'échelon politique. Ce pourquoi aussi, en contexte anglo-américain, Kymlicka n'a pas hésité à théoriser la "citoyenneté multiculturelle» dont la dimension institutionnelle donne lieu à l'éclosion des «droits relatifs à l'au-

8. À ce sujet, les exemples à mobiliser pour s'en convaincre sont légion; simplement pour mémoire retenons les génocides rwandais (près d'un million de morts en I994 et I995) et burundais ainsi que les violences ethniques qui accompagnèrent les élections du 27 décembre 2008 au Kenya et qui ont fait d'après les estimations 3500 blessés, environ 350000 déplacés et I 200 morts. 
tonomie gouvernementale», des «droits polyethniques» et des "droits de représentation spéciaux ${ }^{9}$ ». Indexée à la question de la diversité ethnique dans les États d'Afrique, la trajectoire de la politisation ouvre sur une prise en considération institutionnelle des ethnies dans les mobilisations et répartitions des ressources étatiques.

Faisant suite à une compréhension de la politique comme organisation de la diversité des intérêts en vue d'asseoir harmonie, paix sociale et épanouissement de toute la collectivité, la trajectoire de la "politisation des ethnies» en Afrique est celle des quotas ethniques et de la représentativité dont l'objectif, dans les États multiethniques, est celui d'assurer une répartition équitable des ressources et une participation politique de tous les groupes. On pourra par exemple, sur ce point, se référer aux cas rwandais, burundais et camerounais de la prise en considération politico-institutionnelle des différences ethniques traduite par les «quotas», la «représentation» et la "décentralisation». Dans ces contextes, si la politique des quotas ethniques apparaît comme la réponse à cette exigence de répartir les biens et avantages socio-économiques en fonction de son appartenance à une ethnie, la représentation se traduit par l'exigence de rendre visible (représentation miroir) dans les institutions publiques les différentes identités, et la décentralisation, quant à elle, se laisse appréhender par son souci de résoudre le problème des identités ou des minorités. Pour résumer ces composantes en une seule formule, l'on évoquera sur ce point les termes de l'article $56 \mathrm{du}$ décret $\left(\mathrm{n}^{\circ} 82 / 407\right)$ datant du 7 septembre 1982 au Cameroun:

I. Dans le cadre de l'arrêté portant ouverture de chaque concours administratif d'entrée dans les différentes catégories de la Fonction Publique, le ministre chargé de la Fonction Publique procède à la répartition des places entre les candidats, suivant leurs provinces d'origine, de même qu'il peut réserver des places aux anciens militaires;

3. Le ministre chargé de la Fonction publique fixe par un texte particulier, les quotas de places réservées aux candidats de chaque province, compte tenu de l'importance démographique et du taux de scolarisation de leurs provinces d'origine, et aux anciens militaires sans distinction d'origine.

4. Est considéré comme province d'origine d'un candidat, la province dont ses parents légitimes sont originaires.

L'appartenance ethnique, mise en relief dans le présent décret par la notion d' " origine» des parents, lue avec les trois composantes de la trajectoire de la politisation comblent le souci de reconnaissance et de participation des identités; perspective dont on verra ultérieurement les tenants et les aboutissants à partir d'une analyse plus approfondie. Mais avant d'y parvenir, il importe de rappeler que parler de la politisation des ethnies dans le

9. Cf. la deuxième partie du chapitre II, 200I, p. 44-55. 
champ strictement africain introduit la liaison quotas-représentativité et vient questionner l'approche classique procédant à l'assimilation de ces identités ethniques.

\subsection{Qu'est-ce que privatiser? La trajectoire de la privatisation des ethnies}

Comme on peut le noter à la lumière du développement précédent, la question de savoir comment aborder le fait ethnique des États postcoloniaux en Afrique reste tributaire d'une certaine manière de concevoir la politique en rapport avec sa finalité. L'idée d'une finalité de la politique qui recouvre en ce point celle du comportement nécessaire dans un contexte de pluralisme ethnique "fort» engage donc une compréhension de la politique comme organisation de la société en vue d'un vivre-ensemble harmonieux. Selon cette perspective, le changement de registre qu'introduit la trajectoire de la privatisation tire prétexte d'un contexte de la démocratie s'opérant sur fond de «stérilisation des différences» (Semprini, I997, p. I00) sous les auspices d'un "paternalisme libéral».

L'idée est que, dans son ambition moderne d'instituer une société démocratique juste, la démocratie s'est accommodée de l'égalité, dans l'indistinction. Une égalité comprise comme cécité à l'égard des différences entre les groupes et comme réaffirmation d'un individu pensé en termes d'«être désincarné». À cet égard, un exemple qui pourrait bien être une illustration de cette trajectoire démocratique est la résultante d'une loi du I 3 novembre I97I relative aux Juifs, préparée par la fameuse formule de Clermont-Tonnerre à l'Assemblée constituante le 23 décembre I789: «Il faut tout refuser aux Juifs comme nation; il faut tout leur accorder comme individus; il faut qu'ils soient citoyens ». Dans ces conditions, il devient difficile d'admettre que l'appartenance à un groupe d'origine, fût-il ethnique ou religieux, puisse être le lieu d'une implication du champ politique ou juridique. De nombreuses théories s'emploient sur ce point à conserver l'État hors d'atteinte par les groupes. Il y va d'une «justice dans les institutions ». Par exemple, l' " art de la séparation» que développe Walzer (I997, p. 29-5I) repose en partie sur cette conviction. En effet, selon ce dernier, la démocratie libérale en tant que "justice dans les institutions " pouvait susciter un regain d'intérêt croissant à la faveur de cette séparation entre les sphères: séparation entre l'État et l'Église, séparation entre l'Église d'État et l'Université, séparation de la société civile et de la communauté, séparation de la famille et de l'État, séparation de la vie publique et de la vie privée. C'est en ce sens que pour Walzer (I997, p. 30): "Le libéralisme est un monde de murs $[\ldots] »$.

Dans cet esprit, le mur entre la famille et l'État d'une part, et celui entre la vie publique et la vie privée d'autre part, qui sont les plus en vue dans le cadre de ce travail, ont pour principal avantage de mettre le pouvoir politique à l'abri d'une mainmise interne et de toute tentative d'appropriation de l'État par quelques corps intermédiaires. Dans le courant de ce qui 
s'identifie ici à une séparation entre sphère publique et sphère privée, la question de savoir "qu'est-ce que privatiser?", trouve sa réponse dans l'interdiction de toute référence à des corps intermédiaires au sein de l'État. À preuve la façon dont, dans d'autres débats concernant la catégorisation de l'individu, les instances libérales rejetèrent la référence à une quelconque appartenance jugée «essentialisante ». Nous entendons ici résonner la cloche du féminisme à l'heure de sa critique du libéralisme dans les années I960 aux États-Unis. En effet, durant ces années, le féminisme libéral se donnait pour tâche première d'arracher les femmes à la sphère privée, pour leur redonner accès à l'espace public. L'expérience de ce féminisme et celle de ses prolongements ultérieurs rappellent que la scission public/privé, vecteur cardinal de la pensée libérale, confinait certaines sphères de nos sociétés (la famille et le mariage par exemple) hors du champ du droit et des politiques publiques. De façon typique, la privatisation de l'ethnie peut s'entendre par son exclusion de la vie politique, son exclusion de l'application des lois et, par ricochet, son exclusion de la sphère des politiques publiques. De fait, la référence à la «privatisation» est utilisée pour désigner l'interdiction de l'expression publique des identités ethnico-régionales, religieuses, raciales, perçues comme une contradiction flagrante avec la neutralité, ce principe fondateur de la "communauté des citoyens", pour emprunter une expression à Schnapper (1994).

Dans un registre qui n'est d'ailleurs pas si différent de l'argumentation précédente, la "privatisation » en Afrique, se comprend par rapport à l'exigence d'une "personnalité collective nationale», du moins telle que cette exigence a pu se manifester dans le champ sociopolitique africain scandant l'unité nationale comme horizon de toute politique légitime. Dans ce nouveau climat induit par l'accession à la souveraineté nationale de ces États, la trajectoire d'une "privatisation» en Afrique s'argumente en termes de nécessité d'asseoir une conscience collective nationale, c'est-à-dire de permettre à leurs peuples différents à l'origine de finir par se considérer comme membres indissociables d'une nation «une», «unie» et «indivisible». En termes clairs: «privatiser» prend, dans l'État postcolonial d'Afrique, la trajectoire du discours nationaliste (ainsi que ses effets sur l'organisation politique de ces nations) dont le propos d'Ahmadou Ahidjo, premier président du Cameroun, en est ici l'expression patente:

Nous voulons et nous devons convaincre tous les Camerounais de l'impérieuse nécessité de l'unité nationale... En ce qui nous concerne, dans nos décisions, nous excluons toute considération, tout facteur susceptible de confirmer ou d'entretenir directement ou indirectement les particularismes tribaux... L'unité nationale veut dire qu'il n'y a sur le chantier de la construction nationale ni Ewondo, ni Douala, ni Bamileké, ni Boulou ni Foulbé, ni Bassaà, etc., mais partout et toujours des Camerounais (cité par Mbuyinga, I989, p. 29).

Dans le sens de ce discours qui a fini par concrétiser sur le continent noir le rejet du pluralisme au sortir de la colonisation, la trajectoire de la 
privatisation s'y manifeste par le « rejet », l' « exclusion », la « distanciation » des appartenances, fussent-elles ethniques, régionales, claniques ou tribales, de la sphère publique.

Â tout prendre, la trajectoire de la politisation est celle d'une "visibilisation" institutionnelle de l'origine ethnique, incarnée dans la "politique des quotas» et dans la «représentativité »; et la trajectoire de la privatisation est celle - on pourra en déduire sans coup férir — d'une «invisibilisation» de cette appartenance. Ainsi, "privatiser» devient inéluctablement synonyme de la quête de bonheurs privés alors que "politiser" résonne comme une quête du bien-être et du bonheur collectifs, c'est-à-dire la bona vita. En clair, si la trajectoire de la politisation est cohérente avec le contexte actuel d'effervescence des identités en politique en adhérant à une politique des identités ethniques jugée nécessaire pour la stabilité politique, la trajectoire de la privatisation, elle, ne se soucie nullement de cohérence en promouvant un individu béatement abstrait au rang de fondation ultime d'une démocratie politique juste.

\subsection{Un risque de fragmentation ethnique}

En soi, la trajectoire de la politisation et celle de la privatisation ne posent aucun problème. Ce pourquoi, par exemple, Mbonda (2003, p. I 8) affirme que «la pratique des quotas dans les représentations des groupes peuvent, à cet égard, si elles sont bien appliquées en toute équité, constituer une solution efficace "; à quoi un partisan du libéralisme classique pourrait répliquer que la bonne application des principes individualistes est source de stabilité sociale. Cette double approche nous conduit à un paradoxe qui, quelle que soit l'alternative privilégiée, rend compte de la complexité d'une recherche de la «grande communauté » dans l'État moderne en Afrique. En ce sens, le traitement politico-juridique de la pluralité ethnique par le Rwanda illustre de manière exemplaire le contraste d'une politisation de ces référents ethnoidentitaires.

Le moins que l'on puisse dire sous l'éclairage des arguments historiques est que la mise en application de la politisation peut ouvrir sur un délitement du tissu social. Cela d'autant plus que le critère de la représentativité, tout comme celui des quotas ethniques, peut servir à son contraire, c'est-à-dire à l'affrontement interethnique. À procéder par la traduction concrète de la dialectique quotas-représentativité, on aboutit à l'exacerbation du sentiment ethno-identitaire dont la plus en vue est celle des Tutsi, population estimée jadis à $9 \%$. Ainsi que rapporté par Chrétien (I985, p. I 58 - I 59 ):

D’octobre 1972 à février I973, des bandes Parmehutu entreprirent de vérifier si le taux de $9 \%$ était respecté dans les écoles, faisant expulser les étudiants en surplus, comme si le numerus clausus devait fonctionner mécaniquement de la classe de sixième à la licence. En février-mars I973, des salariés furent chassés de leur emploi pour le même motif. Les violences qui accompagnèrent ce mou- 
vement déclenchèrent une nouvelle vague d'émigration tutsi. L'épuration ethnique alla jusqu'à la chasse aux "hybrides" (ibyimanyi) issus de mariages mixtes et aux «tricheurs» (abaguze ubwoko) qui avaient changé de catégorie raciale!

Faut-il déduire de ce mouvement Parmehutu que l'application des quotas est dans une large mesure vectrice de fragmentation? Cette conclusion apparaît comme la plus conséquente. Et il n'y aurait dans le cadre de son application concrète aucun obstacle à épouser une pareille conclusion. Corrélativement à cette division récurrente des appartenances identitaires induite par la politique des quotas-représentativité, la trajectoire de la privatisation invite dans le même registre à une analyse.

De la même façon, la trajectoire de la privatisation se donne à observer dans le vécu politique contemporain comme une source de désagrégation du lien social. Le processus de privatisation, qui consiste à exclure de toute considération s'exprimant en termes de justice et d'égalité les formes d'appartenance ethnique a été à l'origine de multiples revendications ethnoidentitaires au motif de ce qui leur semble être un déni de reconnaissance empruntant parfois l'adjectif "culturelle ", quand le déni consistait à représenter la "culture de l'Autre" dans sa singularité, comme un domaine séparé du droit. Sous la férule de ces nouvelles formes de revendications inscrites dans le concert de la reconnaissance culturelle, c'est le rapport à la justice qui s'inverse. À cet égard, le «tournant culturel» des sociétés contemporaines, pour parler comme Fraser, féconde de nouvelles revendications de justice qui «ne s'exprimeraient plus uniquement en fonction de principes de redistribution économique, mais emprunteraient également le vocabulaire de la reconnaissance culturelle» (Guéguen, Malochet, 20I2, p. 92). De ce fait, la trajectoire de la privatisation contient les germes de l'exclusion quotidienne et celle d'une discrimination économique, sociale et politique à l'égard de certaines communautés se trouvant dans l'espace étatique. On pourra d'ailleurs, pour en être convaincu, se référer aux récentes revendications de certaines minorités dites «visibles» qui se sont retrouvées dans la posture des «exclues» de la République au nom d'un crédo républicain obnubilant les différences entre les communautés. La multiplication croissante de ces revendications dans les États démocratiquement avancés fait apparaître aujourd'hui l'idéal d'une nation béatement abstraite comme relevant d'une extrême curiosité.

Dans le contexte africain, la trajectoire de la privatisation de l'ethnie qui tirait justification de la nécessité, au lendemain de la colonisation, de construire des nations politiques prospères donna lieu à l'institution d'États communautaires. L'État communautaire, nous dit Le Bot (I997, p. I 87), "cherche à expurger la société des éléments d'hétérogénéité, à rétablir ou repousser la frontière, à "nettoyer" le territoire, à réaffirmer l'identité, par une violence qui, en réalité, réactive et approfondit les déchirures ". "Volonté d'homogénéisation » à travers cette "pulsion de destruction de l'Autre inté- 
rieur ", affaiblissement puis décomposition de l'État dus aux affrontements ethniques nous servent à décrire le relief d'un État communautaire. On se souvient de ce qu'a engendré la politique d'édification d'un État communautaire en Haïti par le président Aristide au cours de son premier passage à la tête de cet État en I99I. On se souvient dans le même creuset que l'idéologie de l'unité nationale, s'affirmant hostile à l'égard de tout particularisme au sein des frontières stato-nationales africaines, fit bon ménage avec de réelles injustices subies par beaucoup de groupes dans la répartition et dans l'accès aux ressources de l'État. Le cas de la revanche Hutu contre la communauté Tutsi à partir de la venue au pouvoir de Grégoire Kayibanda transformant l'État communautaire en un champ de bataille entre les différentes communautés ethniques en témoigne suffisamment.

Dans ce contexte, la métaphore de la boîte de Pandore suggère que «politiser» ou "privatiser» ne peut en aucune manière être la condition sine qua non d'une participation authentique à la constitution du bien commun. Ainsi l'ethnisme devient inéluctable à partir du moment où il se trouve figé une fois pour toutes tant dans une trajectoire politique que dans une trajectoire privée: le grief que l'on pourrait faire à la trajectoire de la politisation est celui d'une "essentialisation" du pôle ethnique, et à la trajectoire de la privatisation, celui de l'affirmation du pôle ethnique comme résultat d'une « instrumentalisation ». Dans ces conditions, il devient légitime de se demander à quoi pourrait s'identifier véritablement la valorisation du pluralisme ethnique. Ainsi sommes-nous portés à nous interroger: comment aborder désormais le fait ethnique d'Afrique?

\section{L'horizon de la postcommunauté comme artéfact du bien commun à l'âge postcolonial}

Dans Politiques (Livre I, chap. II, I983, p. 27-30), Aristote suggérait déjà qu'il faut dépasser la famille, le village, pour la Cité. Contre la logique de quoi, à beaucoup d'années de distance entre Aristote et l'institution de l'État en Afrique, l'affirmation des communautarismes se trouva associée au nom d' "ethnisme » qui exprime la difficulté du citoyen africain à solder l'héritage colonial, durablement inscrit dans ses structures mentales, psychiques et légales, pour espérer s'accommoder avec la perspective aristotélicienne. Cette difficulté s'évalue par comparaison avec les multiples réaffirmations ethno-identitaires se dressant contre l'État-nation en Afrique. Comme par voie de conséquence, le préfixe "post» dans la "postcommunauté » nécessite un double cadrage: d'un côté, il n'implique pas une cécité des différences entre les communautés ethniques, et de l'autre, il ne se réduit pas à l'horizon étroit de la simple politisation des identités.

Dans le premier cas concernant l'indifférence à l'égard des appartenances ethno-identitaires, cette attitude que l'on doit en partie à la trop grande place accordée à l'individu par les adjuvants d'un "paternalisme libéral» est irrecevable, quel que soit le sens attribué à la postcommunauté. 
Car il n'est certainement pas vrai que la remise en cause des communautés que résume le mot "privatisation " parvient véritablement à reléguer dans quelque préhistoire politique la référence à des identités ethniques. Dans le second cas, la simple reconnaissance institutionnelle des appartenances ethno-identitaires reviendrait à occulter la postcommunauté des questionnements toujours récurrents en dépit de la politisation, comme si d'ailleurs l'institutionnalisation des identités que traduit le mot "politisation " implique réellement une unité quasi fusionnelle des intérêts à l'échelle ethno-identitaire pensée éventuellement comme un havre de paix et de protection abritant un type particulier de relations humaines homogènes. Précédemment, l'on a décelé dans chacune des deux options de cette alternative une recette d'instabilité sociale. En conséquence, ainsi que nous le montrerons dans les lignes à suivre, la postcommunauté ne signifie pas négation (au sens premier du mot) de la communauté, mais son inclusion non "essentialisante» dans un nouveau processus d'édification du bien commun dans l'État en Afrique. Démarche que résume parfaitement la dialectique hégélienne en termes d'affirmation, de négation puis de négation de la négation. Dans cet esprit, nous aborderons les questions, d'abord de la conscience (individuelle et collective), puis de la participation des identités ethniques à la construction du «commun", enfin de la responsabilité de l’État.

\subsection{La part de la conscience dans l'édification du bien commun en Afrique}

Comme le recommande l'Unesco pour ce qui est de la paix: "La guerre prenant naissance dans l'esprit des hommes, c'est dans l'esprit des hommes que doivent être élevées les défenses de la paix ${ }^{10}{ }$. Nous pouvons reprendre en la modifiant légèrement cette recommandation de l'Unesco: "C'est dans la conscience des hommes que se joue durablement l'ethnisme, c'est dans la conscience des hommes que doivent être ôtées les racines de l'ethnisme ». Ce constat prend de l'ampleur quand on se rappelle que la trajectoire de la politisation donnait lieu, ainsi que nous le discutions en amont, à une série de mesures politico-juridiques servant de balises à l'expression effrénée du pluralisme ethnique mais que dans le même temps, malgré ces balises juridiques, les identitarismes ethniques persistent toujours comme des pratiques rebelles.

De fait, ce qu'il nous semble important de mettre en relief réside justement dans l'impossibilité de considérer l'institutionnalisation des différences ethniques comme une fin en soi étant donné que cette institutionnalisation n'exclut pas dans le même temps une mainmise des gouvernants sur toutes les sphères par le truchement d'une forte concentration du pouvoir. Ainsi devient-il impérieux que conscience soit prise sur tous les plans: aussi bien sur le plan individuel que sur le plan des différentes collectivités, puisque

10. Préambule de la Convention constitutive, Textes fondamentaux de l’Unesco, édit. $20 \mathrm{I} 2$. 
l'ethnisme devient inéluctable en dehors de l'injonction éthique qui, au-delà de la loi, fait appel à la conscience de chaque personne. Dans des termes éthiques, il s'agit des personnes dont la conscience est encline au respect des lois; ce qui empêche ainsi toute logique d'instrumentalisation des textes en vigueur. On peut considérer que, même exprimée dans un système juridique effectif et par des actions politiques concrètes, la gestion de la diversité, particulièrement de la diversité ethnique en Afrique postcoloniale, requiert de manière prioritaire une intériorisation dans la conscience de chaque citoyen. Car, comme le rappelle Renaut (2009, p. 427):

[...] dans l'espace propre de l'éthique, c'est le sujet moral qui, au-delà de tels apprentissages, ne peut ultimement que s'obliger lui-même à souscrire aux principes éthiques de son existence, ce sujet m'apparaît [...] l'acteur indépassable qui décide de la place tenue dans sa vie par sa perception de la diversité humaine.

C'est donc admettre que l'élaboration des lois ne saurait suffire pour réaliser l'objectif escompté qui est celui de la pacification des rapports humains prédisposés aux conflits en raison de la diversité des intérêts qui passionnent tous les acteurs sociaux. En ce sens donc:

La participation aux biens collectifs, participation consistant non pas en jouissance matérielle, mais en un sentiment de propriété, est un besoin non moins important. Il s'agit d'un état d'esprit plutôt que d'une disposition juridique. Là où il $\mathrm{y}$ a véritablement une vie civique, chacun se sent personnellement propriétaire des monuments publics, des jardins, de la magnificence déployée dans les cérémonies [...] Mais ce n'est pas seulement l'État qui doit fournir cette satisfaction, c'est toute espèce de collectivité (Weil, I949, p. 32).

Cette formule de Weil ouvre, primo, sur la nécessité d'une décolonisation des consciences à l'égard de certains stéréotypes ethno-identitaires confinant le bien public dans la sphère des appartenances ethno-identitaires, et surtout comme un processus censé accompagner les balises juridiques en matière de gestion de la diversité; secundo, sur la nécessité d'un travail d'appropriation du bien de l'État comme une propriété privée et une propriété collective tant cette appropriation se révèle être le signe d'une adhésion de chaque membre à l'œuvre tout entière de la collectivité; tertio, sur une nouvelle forme de participation démocratique des différentes communautés ethniques à l'édification du bien commun dans la figure de l'État en Afrique.

\subsection{La part de la communauté ethnique}

Les regroupements ethniques à l'œuvre de plus en plus dans les espaces publics, symboles de la chose la mieux partagée au sein des États africains, invitent à une nouvelle forme de participation démocratique dans la constitution du commun. En effet, la tâche de construction du bien commun doit prendre en considération la responsabilité des communautés ethniques revendiquant de plus en plus l'accès à une dimension de l'espace public 
qu'illustrent ces regroupements dans les universités, les communes, les préfectures, etc. Dans ce schéma, la conscience ethnique ne peut véritablement être «dépassée » que si les membres des différentes communautés ethniques participent à la construction du bien commun dans l'État postcolonial. À cet effet, le trio conceptuel de Zask, qui incarne désormais les conditions d'une participation authentique: "prendre part», "contribuer" et "bénéficier», nous paraît intéressant.

La publication du livre, Participer. Essai sur les formes démocratiques de la participation, a comme objectif chez son auteure d'analyser les significations et valeurs philosophiques fondamentales inhérentes à la participation. Pour ce faire, Zask opère, dans le premier échelon du trio, une distinction entre "prendre part» et "faire partie». Tandis que «faire partie» renvoie dans la logique de cette auteure à la simple appartenance à un groupe donné, "prendre part» indique la nécessaire implication dans la production du bien commun envisagé désormais comme une "production", et non comme un "héritage». De même que «les randonneurs décident de leur itinéraire, d'un point de rendez-vous, d'un horaire, d'un but précis [...]", de même, écrit Zask (20I I, p. 44) quelques lignes auparavant, «[...] le "prendre part" est par ailleurs une configuration où les participants d'un côté et les activités auxquelles ils participent de l'autre sont conditionnés mutuellement et ne peuvent être séparés". Ainsi donc: "S'associer ne signifie donc pas partager un bien commun, mais produire en commun quelque chose qui, ultérieurement et de diverses façons, est apprécié par chacun des participants et s'offre à lui (prendre une part) comme une ressource supplémentaire d'individuation» (Zask, 20II, p. 89). De ce fait, nous voulons admettre que le défi toujours renouvelé du bien commun à la pluralité ethnique dans les États africains reste tributaire d'un manque de repères communs à la production desquels auraient participé les différentes collectivités revendiquant chacune une part de l'État ou dans une large mesure tout l'État. La réalité étant par exemple qu'au sortir de la colonisation le constitutionnalisme moderne en Afrique s'est trouvé et se trouve encore happé par les questions d'autochtonie et de minorités ethniques marginalisées.

De surcroit, il nous semble important de noter que la thèse de l'instrumentalisation des ethnies, thèse qui jouit d'une popularité assez forte dans le milieu politique comme dans le milieu intellectuel, se trouve érodée à la lumière du simple «faire partie» qui, dans le contexte de la pluralité ethnique, se borne à une dimension de la socialité comme intégration des communautés ethniques dans des États déjà constitués, et une autre dimension de la socialité comme articulant ces différentes communautés autour d'un bien commun non à construire mais supposé préalablement défini. Pour le redire en termes plus clairs: c'est pour nous l'absence du bien commun à la construction duquel auraient dû participer l'ensemble des communautés ethniques de l'État postcolonial qui favorise cette logique instrumentale et mobilisatrice de la fibre ethnique. Car être manipulé est une chose, se laisser 
manipuler en est une autre. En conséquence, il en résulte que les différentes composantes ethno-identitaires de l'État se laissent manipuler, faute d'une connaissance claire de la nécessité d'un bien émanant de la solidarité organique: "De lui-même le peuple veut toujours le bien, mais de lui-même il ne le voit pas toujours" (Rousseau, I966, p. 76). D'où découle alors l'évidence que la pratique mobilisatrice et calculatrice des identités ethniques trouve son fondement dans l'absence d'un bien commun "construit» puis "partagé » par tous les membres de ces différentes communautés en tant que "bien suprême". Car "c'est en vue de ce qui leur semble le bien que tous les hommes font tout ce qu'ils font" (Aristote, I983, p. 26). De ce fait, reprenant d'assaut l'urgence d'une transition du « faire partie » au "prendre part", ladite transition fait désormais appel à «[...] une philosophie publique ou conversation entre concitoyens" (Tully cité par May, 20I6, p. I70) à l'image d'un dialogue permanent autour du "commun" entre universitaires, "hommes de décrets", citoyens ordinaires, tous membres de différentes communautés ethniques posées au départ du dialogue comme égales.

Cette perspective offre les clés d'une «contribution» des membres des différentes communautés ethniques: "participer», c'est alors «contribuer». La contribution sous la plume de la disciple de Dewey, Zask, se laisse appréhender comme l'apport multiforme des hommes à leur groupe d'appartenance. C'est donc une tâche de "coopération" qui consiste pour les êtres humains à apporter «[...] à leur groupe des éléments en propre qu'ils peuvent percevoir comme la preuve ou la marque de leur existence sociale, et qui favorisent l'évolution du groupe vers une adaptation et une recomposition du commun [...]» (ibid., p. I 53). Dans cette multiformité, la contribution des différentes composantes ethniques peut consister par exemple à se départir, sur le plan communautaire, de la référence au "garant métapolitique» (Touraine, I994, p. IOI) qui renferme des catégories non politiques (la race, le dieu, la langue, etc.), lesquelles sapent durablement la naissance d'un esprit public.

Sous cet angle, il convient de dire que les membres des différentes communautés ethniques ne sauraient participer véritablement à la production du «commun» que s'ils jouissent réellement d'un environnement favorable à la délibération, c'est-à-dire d'un environnement non défaillant que pourrait représenter par exemple l' «individualisme» ou celui plus étouffant que pourrait constituer une "religion républicaine». Cette précision ouvre donc la voie à la troisième dimension de la participation: "participer ", c'est aussi "bénéficier». "Bénéficier ", c'est "recevoir une part» ou, en suivant le registre nominal, le "bénéfice », comme dit l'auteure, est "un élément doté de qualités lui permettant d'entrer dans une combinaison avec celles $d u$ bénéficiaire [...] » (ibid., p. 236). Le souci du bénéfice vers lequel converge en dernier ressort la pensée de Zask interpelle l'État en Afrique. 


\subsection{La part de l'État}

On affirme aujourd'hui que la démocratie en Afrique doit être à l'image de ses États formés d'une mosaïque d'ethnies rassemblées dans un même creuset au gré de l'histoire colonisatrice. Dans le sens de cet aveu, aborder le fait ethnique de l'Afrique postcoloniale rappelle en premier cette jonction entre les deux critiques communautariennes du libéralisme: "[...] l'État n'est pas, en fait, l'unique, ni même, pour les gens ordinaires dans leur vie de tous les jours, la plus importante des unions sociales" (Walzer, I997, p. 73). L'existence d'autres unions sociales (telles que les ethnies, les clans, les tribus, etc.) avec une conscience exacerbée au sein de l'État s'illustre dans le contexte africain par une sorte d' «imposture ethnocentriste» et le vote régionaliste, deux moyens par lesquels les différentes unions sociales (ethniques) au sein de l'État moderne en Afrique tentent de s'accaparer la sphère du public. Cet accaparement de l'État en Afrique par des communautés ethniques, plutôt que d'être appréhendé comme la résultante d'une instrumentalisation à des fins politiques, est plutôt lu ici comme découlant d'une dimension du bien défini par la "solidarité mécanique " liée donc à une absence notoire de valeurs communes «construites» puis "partagées» par toutes les communautés ethniques réunies au sein des nouvelles frontières étatiques.

Dans cet univers régi par la "solidarité mécanique ", où la propension de tout un chacun à faire ce qu'il fait en fonction de ce qu'il entend par «le bien » pouvant dégénérer en une "guerre des dieux ${ }^{11}$ ", s'impose la nécessité pour l'État de redéfinir de nouveaux liens sociaux. En effet, s'il s'avère évident de lire la bigarrure ethnique et son symposium avec les lunettes de cette jonction communautarienne, le moins que l'on puisse en dire est qu'il devient impérieux pour l'État africain de redéfinir de nouvelles valeurs aptes à inscrire les préoccupations de chaque groupe ethnique dans le creuset national. À cette fin, nourri par la passion de ne léser aucune communauté ethnique, qu'elle appartienne à la minorité ou à la majorité, ou qu'elle soit de l'opposition ou de la mouvance politique, il importe de recourir à une «éthique procédurale» dans la définition de ce bien commun qui doit régir la vie de tous les citoyens sur le plan politique.

Encore faudrait-il souligner qu'une définition procédurale du bien commun peut sembler aussi inopérante, dans la mesure où rien de tout cela ne garantit contre l'érosion de nouveaux liens sociaux ou contre la mort des devoirs envers sa communauté d'origine. Ce constat prend toute son ampleur quand on se rappelle que, dans les États où l'on a procédé à une reconnaissance institutionnelle de l'ethnie incarnée dans des textes, puis traduite dans la réalité, il y a eu également un problème de cohésion sociale. Cela correspond à une recherche de liaison entre justice et bien commun. D'où découle

11. Laquelle guerre met à rudes épreuves le bien commun comme aspiration collective de tous. Sur cette question du bien commun et de son rapport à la "guerre des dieux ", nous renvoyons le lecteur à la première partie de l'article de Mbonda, 20I I, p. I I4-I I 8. 
la nécessité d'enjoindre à l'édification du bien commun comme bien souverain une liaison à la justice. En ce sens par exemple, lorsqu'en $20 \mathrm{I} 7$ les Tem se soulevèrent au Togo pour dénoncer l'illégitimité d'un chef de canton Kabyè sur leur territoire (celui de Lama-Tessi en l'occurrence), c'est à l'idée du «J'étais-là avant» dans son versant contemporain du "Ôte-toi de là que je m'y remette ", qu'un tel soulèvement faisait explicitement référence. Dans le cadre de ce soulèvement, participant à la problématique de l'autochtonie, la responsabilité première est celle de l'État porté par les "hommes de décrets» qui se trouvent sommés de prendre des dispositions urgentes pour restaurer la partie de la République lésée dans ses droits et pour empêcher que cette revendication ne dégénère en conflit ethnique.

Dans cet appel à la responsabilité des gouvernants, l'idéal de justice à promouvoir ne nous semble pas être celui de la "politique des quotas" fixant une fois pour toutes la répartition des rôles selon des critères ethniques déterminés. Le fait est qu'en assujettissant la participation politique à des critères ethniques déterminés une fois pour toutes risque de former un «assemblage de ghettos ethniques » que résume proprement le terme de «communautarisme» entendant "[...] l'enfermement des personnes dans un groupe particulier, aux dépens de leur conscience commune et de leurs relations, au-delà de leur groupe, avec les membres de la société plus large» (Schnapper, 2004, p. г80). L'orientation qui sous-tend la trajectoire d'une "politique des quotas ${ }^{12}$ " ethniques est à notre humble avis

12. La «politique des quotas" telle qu'elle apparaît à ce niveau peut induire deux éventuelles objections: I) l'une, consistant à affirmer que la politique des quotas pourrait aussi être considérée comme relevant de l' "expérimentation ", c'est-à-dire un dispositif visant à répondre à des formes précises de revendications et non comme une fin en soi (telle la "politique de discrimination positive » aux USA et en Afrique du Sud passant pour une mesure transitoire de «justice correctrice»); 2) l'autre, affirmant que la politique des quotas n'a pas à obéir à un principe de justice statistique ou mathématique (c'est-à-dire en tenant compte de la taille démographique de chaque groupe), mais à un principe de justice politique répondant à la nécessité de faire participer chaque groupe. À chacune de ces deux éventuelles objections nous voudrions, respectivement, répondre: I) la conception de la politique des quotas telle qu'elle apparaît dans le corps de ce texte fait suite à la position de certains " africanistes " plaidant en faveur de la politique des quotas ethniques comme un fondement nec plus ultra dans l'État postcolonial. L'exemple de Mwayila Tshiyembé (2005, p. 33-40), (200I), chez qui la politique des quotas n'est nullement une «expérimentation» mais une exigence inéluctable dans l'État postcolonial en Afrique; 2) nous nous bornerons à rappeler que, dans l'État postcolonial d'Afrique, cette nécessité de faire participer tous les groupes, quand elle s'est traduite dans des dispositifs politiques concrets, a fini par consacrer l'émiettement de certains groupes en plusieurs sousgroupes donnant ainsi lieu à une multiplication croissante des clivages au sein de groupes que l'on souhaitait faire participer. Par exemple, sous l'effet de la Population Registration Act en I950, l'afrikaner qui avait à l'esprit le rétablissement de la justice entre deux populations, les Blancs et les Noirs (ces derniers ayant pendant longtemps été marginalisés), s'est confronté à une division continue de la population noire (que les politiques en place voulaient "faire participer») en neuf ethnies (les Xhosas, les Zoulou, les Tswanas, etc.), qui seront à leur tour subdivisées suivant des critères ethnolinguistiques plus restreints. En conséquence de quoi cette nécessité de faire participer chaque groupe, conformément aux exigences de la justice politique, 
sans lendemain ${ }^{13}$. Nous sommes d'accord avec Semprini qui, dans Le multiculturalisme ( 1997, p. 76) rapportait que ces politiques fondées sur des critères rigides avaient fini par véhiculer chez la population issue de la race noire aux États-Unis des "stéréotypes et des préjugés". On se rappelle à ce sujet avec Dahl (I998, p. I89) que ces politiques "qui avaient provisoirement apporté la stabilité au Liban, au Nigéria et au Sri Lanka, n'ont pas résisté à la violence des conflits ethniques ni pu éviter que ces pays connaissent la guerre civile [...]». Au constat de ce propos, nous voulons plaider pour une "justice compensatrice", laquelle apparaît sous la plume de Renaut (2007, p. I2I) comme une "justice correctrice» ou "justice réparatrice" fondée sur le principe: "à chacun ce qui lui revient en fonction de ce dont il a été privé ou spolié». Il y a lieu en ce point précis de faire appel à la notion de "politique comme une "expérimentation" (Dewey, 20I5[I9I5], p. II) et non plus comme une "conformité à des principes intangibles ".

\section{Conclusion}

Du fait de sa force communalisante et socialisante, l'ethnie oscille depuis toujours entre une politique d'assimilation et sa naturalisation normative d'une part, et son instrumentalisation, d'autre part. Consécutivement à une Afrique traditionnelle au sein de laquelle le bien du collectif primait sur celui de l'individu, on se serait attendu à ce que l'ère postcoloniale soit le lieu d'établissement de nouveaux repères prompts à propulser la «nation" moderne en Afrique vers une conscience plus aiguë du bien commun. A priori, l'on se serait attendu également à une "participation» effective des différents membres de ces communautés ethniques à l'établissement du bien commun compris au sens aristotélicien comme «le bien suprême », le souverain bien ou le summum bonum suivant la terminologie latine. Mais au contraire, une nouvelle pratique politique, la «cooptation », rendue possible par la nouvelle «socialisation politique occidentale» des indigènes vient témoigner de l'exacerbation de la conscience ethnique. Cette situation fait alors que "L'ethnie est perçue à la fois comme le "refoulé" et le "revenant" en regard de la pratique sociopolitique» (Akono Evang, 20I4, p. I72). «Refoulé », dans le contexte politique et scientifique de la construction de la nation postindépendance; "revenant ", dans le contexte de démocratisation qui consacre une montée fulgurante des revendications ethno-identitaires.

peut aussi générer une "ghettoïsation » des différentes communautés que la présente contribution ambitionne de mettre en abîme.

13. Analysée en profondeur, l'absence de données statistiques indiquant avec précision le nombre de membres composant chaque groupe ethnique constitue, à l'état actuel, un obstacle majeur à la mise sur pied d'une telle politique en Afrique. Encore faut-il préciser que, jusque dans les pays développés où ces données statistiques existent déjà, la politique des quotas n'a encore pas fini de révéler ses dessous inquiétants pour ce qui est de la désaffection sociale, de la remise en cause du principe démocratique du mérite. 
Ces dernières allant jusqu'aux formes les plus «extrêmes» de leur expression que constituent l'épuration ethnique et le génocide. Cette errance du fait ethnique entre «refoulement» et «institutionnalisation» est l'indice de la pertinence d'une réflexion portant sur la "politisation" et la "privatisation» de l'ethnie dans la poursuite du bien commun. À cet égard, l'objet du présent article est de situer les limites de la trajectoire de la politisation et de celle de la privatisation. En effet, certaines des difficultés philosophicopolitiques que pose la recherche du bien commun dans l'État postcolonial en Afrique se trouvent aussi bien à l'échelle de la politisation qu'à l'échelle de la privatisation de l'ethnie: d'une part, parce qu'il y est question dans les deux cas d'un risque d' "explosion ethnique nucléaire", lié soit à une "stérilisation" des différences ethniques, soit à une "institutionnalisation" de ces différences, ou encore à l'impossibilité de pacifier le «droit à la différence»; d'autre part, parce qu'on peut considérer que l'inégalité des rapports de force qu'on observe entre les différentes ethnies au sein des États postcoloniaux d'Afrique est un résidu de déstabilisation. Il reste toutefois légitime et éclairant de mobiliser un nouveau cadre normatif pour réfléchir aux enjeux philosophico-politiques que soulèvent la politisation et la privatisation des ethnies en Afrique. L'objet de cet article est in fine de mobiliser le cadre théorique et pratique de la post-communauté.

\section{Bibliographie}

Akono, Evang, Serge Paulin. "Contribution à une science africaine de l'ethnie à partir de l'expérience camerounaise ", Droit et société, n 86, 2014, p. I 57I 74 .

Amselle, Jean-Loup. Logiques métisses. Anthropologie de l'identité en Afrique et ailleurs, Paris, Payot, I990.

Amselle, Jean-Loup, et Elikia M'bokolo. 1985. Au cour de l'ethnie. Ethnies, tribalisme et État en Afrique, Paris, La Découverte, I 999.

Aristote. Politiques, Livre I, trad. Pellegrin Pierre, Paris, Éditions Nathan, I983.

Bayart, Jean-François. L'État en Afrique. La politique du ventre, Paris, Fayard, I989.

Bayart, Jean-François, et Peter Geschiere. «"J'étais là avant". Problématiques politiques de l'autochtonie", Critique internationale, $\mathrm{n}^{\circ}$ I0, 200I, p. I26-I 28.

Bazin, Jean. I985. "À chacun son Bambara", in Jean-Loup Amselle et Elikia M'bokolo, Au cour de l'ethnie. Ethnies, tribalisme et État en Afrique, Paris, La Découverte, I999, p. 84-I 27.

Bidima, Jean-Godefroy. "Le corps, la cour et l'espace public», Politique africaine, $\mathrm{n}^{\circ} 77,2000$, p. 90-106.

Chrétien, Jean-Pierre, et Gérard Prunier. Les ethnies ont une histoire, Karthala, I989.

Dahl, Robert. De la démocratie, Paris, Economica, I 998.

Dewey, John. I91 5. Le public et ses problèmes, Paris, Gallimard, 2015.

Dijon, Xavier. "Les masques du droit en Afrique. Une relecture européenne des sources de la norme", Revue de droit international et de droit comparé RDIDC, $\mathrm{n}^{\circ} 4,2009$, p. 567-60I. 
Dijon, Xavier, et Marcus Ndongmo. L'éthique du bien commun en Afrique. Regards croisés, Paris, L'Harmattan, 20I I.

Dorier-Apprill, Elisabeth. "Géographie des ethnies, géographie des conflits à Brazzaville », in Patrice Yengo (dir.), Identités et démocratie, Paris, L'Harmattan, I997, p. I 59-I 86.

Freund, Julien. Qu'est-ce que la politique? Paris, Sirey, 1965.

Guéguen, Haud, et Guillaume Malochet. Les théories de la reconnaissance, Paris, La Découverte, 2012.

Habermas, Jürgen. L'Espace public, Paris, Payot, I986.

Kant, Emmanuel. Critique de la raison pure, trad. Alain Renaut, Paris, GFFlammarion, 2006.

Kiamba, Claude-Ernest. «Le bien commun en Afrique: entre tradition et modernité ", in Xavier Dijon et Marcus Ndongmo, L'éthique du bien commun en Afrique. Regards croisés, Paris, L'Harmattan, 20I I, p. I9-34.

Kymlicka, Will. La citoyenneté multiculturelle. Une théorie libérale du droit des minorités, trad. Patrick Savidan, Paris, La Découverte, $200 \mathrm{I}$.

Le Bot, Yvon. "Le temps des guerres communautaires », in Michel Wieviorka (dir.), Une société fragmentée? Le multiculturalisme en débat, Paris, La Découverte, I997, p. I73-I97.

Mbembe, Achille. De la postcolonie. Essai sur l'imagination politique dans l'Afrique contemporaine, Paris, Éditions Karthala, 2000.

Mbonda, Ernest-Marie. "Bien commun et éthiques procédurales », in Xavier Dijon et Marcus Ndongmo, L'éthique du bien commun en Afrique. Regards croisés, Paris, L'Harmattan, $20 \mathrm{II}$, p. II I-I 24.

—. "La "justice ethnique" comme fondement de la paix dans les sociétés pluriethniques. Le cas de l'Afrique", in Josiane Boulad-Ayoub et Luc Bonneville, Souverainetés en crise, Québec, L'Harmattan et les Presses de l'Université Laval, 2003, [p. 45I-500], 57 p.

Disponible sur le site: http//www.uqac.ca/Classiques_des_sciences_sociales/

Mbuyinga, Elenga. Tribalisme et problème national en Afrique noire. Le cas $d u$ Kamerun, Paris, L'Harmattan, I989.

Nicolas, Guy. «Les Nations à polarisation variable et leur État: le cas nigérien », in Emmanuel Terray (dir.), L'État contemporain en Afrique, Paris, L'Harmattan, I987, p. I 57-I74.

Otayek, René. "L'Afrique au prisme de l'ethnicité: perception française et actualité du débat ", Revue internationale et stratégique, $\mathrm{n}^{\circ} 43$, 200I, p. I29-I42.

Rawls, John. Justice et démocratie, Paris, Seuil, I993.

Renaut, Alain. Un humanisme de la diversité. Essai sur la décolonisation des identités, Paris, Flammarion, coll. "Bibliothèque des savoirs ", 2009.

Égalité et discriminations, Paris, Seuil, 2007.

Rousseau, Jean-Jacques. Du contrat social, Paris, GF-Flammarion, I996.

Schnapper, Dominique. "La République face aux communautarismes", Études, tome 400, 2004, p. I77-I 88.

—. La communauté des citoyens, Paris, Gallimard, I994.

Semprini, Andrea. Le multiculturalisme, Paris, PUF, "Que sais-je ? ", I997.

Sindjoun, Luc. La politique d'affection en Afrique noire, Publications du GRAF, Boston, Boston University, I998. 


\section{Philosophiques / Automne 2018}

Taylor, Charles. Multiculturalisme. Différence et démocratie, trad. Canal DenisArmand, Paris, Aubier, I994.

Touraine, Alain. Qu'est-ce que la démocratie?, Paris, Fayard, I994.

Tshiyembé, Mwayila. "État multiethnique en tant que modèle de nouvelle gouvernance en Afrique noire de ce début du XXI ${ }^{\mathrm{e}}$ siècle ", Présence africaine, $\mathrm{n}^{\circ} \mathrm{I} 75^{-}$ I76-I77, 2007, p. 34I-353.

—. «Ethnies: restons aussi nous-mêmes!», Outre-Terre, vol. 2, n II, 2005, p. $33-40$.

—. État multinational et démocratie africaine. Sociologie de la renaissance politique, Paris, L'Harmattan, 200I.

Walzer, Michael. Pluralisme et démocratie, Paris, Esprit, I997.

Weber, Max. Economie et société, t. 2, Paris, Plon, 2003.

Weil, Simone. L'enracinement. Prélude à une déclaration des devoirs envers l'être humain, Paris, Gallimard, I949.

Disponible sur le site: http//www.uqac.ca/Classiques_des_sciences_sociales/

Zask, Jöelle. Participer. Essai sur les formes démocratiques de la participation, Paris, Le Bord de l'eau, 20I I. 